\title{
INNER AMENABLE LOCALLY COMPACT GROUPS
}

\author{
ANTHONY TO-MING LAU AND ALAN L. T. PATERSON
}

\begin{abstract}
In this paper we study the relationship between amenability, inner amenability and property $P$ of a von Neumann algebra. We give necessary conditions on a locally compact group $G$ to have an inner invariant mean $m$ such that $m(V)=0$ for some compact neighborhood $V$ of $G$ invariant under the inner automorphisms. We also give a sufficient condition on $G$ (satisfied by the free group on two generators or an I.C.C. discrete group with Kazhdan's property $T$, e.g., $\operatorname{SL}(n, \mathbb{Z}), n \geq 3$ ) such that each linear form on $L^{2}(G)$ which is invariant under the inner automorphisms is continuous. A characterization of inner amenability in terms of a fixed point property for left Banach $G$-modules is also obtained.
\end{abstract}

\section{INTRODUCTION}

Let $G$ be a locally compact group. Then $G$ is called inner amenable if there exists a state $m$ on $L^{\infty}(G)$, such that $m(\pi(a) f)=m(f)$ for all $a \in G$ and $f \in L^{\infty}(G)$, where

$$
\pi(a) f(x)=f\left(a^{-1} x a\right), \quad x \in G .
$$

Amenable locally compact groups and $[I N]$-groups are inner amenable. The group $G$ is [IN] if there exists a compact neighborhood $V$ of the identity $e$ in $G$ such that $a^{-1} V a=V$ for all $a \in G$. Furthermore when $G$ is connected, then $G$ is amenable if and only if $G$ is inner amenable (see [17]). A recent account of amenability is given in [21].

Let $\mathscr{M}$ be a von Neumann algebra on a Hilbert space $H$ and let $\mathscr{M}^{\prime}$ be the commutant of $\mathscr{M}$. For $T \in \mathscr{B}(H)$ (the space of bounded linear operators on $H$ ), let $C_{T}$ be the weak*-closed convex subset of $\mathscr{B}(H)$ generated by $\left\{U^{*} T U ; U \in \mathscr{M}^{u}\right\}$, where $\mathscr{M}^{u}$ is the group of unitary elements in $\mathscr{M}$. (Note that $\mathscr{B}(H)$ has a unique predual $[28$, p. 47].) $\mathscr{M}$ is said to have property $P$ if $C_{T} \cap \mathscr{M}^{\prime} \neq \varnothing$ for each $T \in \mathscr{B}(H)$.

Let $\operatorname{VN}(G)$ denote the von Neumann algebra on $L^{2}(G)$ generated by $\left\{l_{x} ; x\right.$ $\in G\}$ where $l_{x} h(t)=h(x t), t \in G$. A well-known result of Schwartz [29] asserts

Received by the editors April 12, 1989.

1980 Mathematics Subject Classification (1985 Revision). Primary 43A07.

Key words and phrases. Amenable locally compact groups, inner amenable groups, [IN]-groups, property $P$, von Neumann algebra, free group, Kazhdan's property $T$, automatic continuity, fixed point property.

The first author's research was supported by an NSERC grant. 
that if $G$ is discrete, then $G$ is amenable if and only if $\operatorname{VN}(G)$ has property $P$. In $\S 3$ we study the relation between amenability, inner amenability, and property $P$ of a von Neumann algebra determined by $G$ and its action on a locally compact Hausdorff space $X$. In particular, we provide the missing link in the following well-known implications for a locally compact group $G$ :

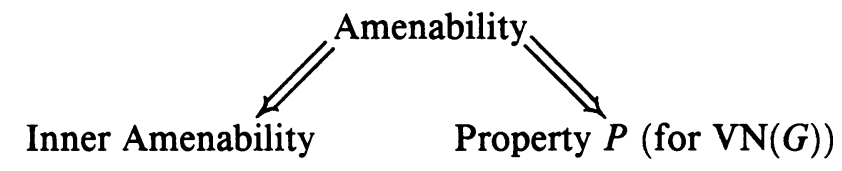

.In [20] Paschke proved that if $G$ is an infinite discrete group, then there exists an inner invariant mean on $l^{\infty}(G)$ different from the point evaluation at the identity if and only if the $C^{*}$-algebra generated by the unitaries on $l^{2}(G)$ corresponding to conjugation by elements in $G$ does not contain the projection on the space $\mathbb{C} \delta_{e}$, where $e$ is the identity of $G$. In $\S 4$, we find necessary conditions for there to exist an inner invariant mean $m$ on $L^{\infty}(G)$ such that $m\left(1_{V}\right)=0$ (when $V$ is a compact neighborhood of $G$ invariant under inner automorphisms). We also give a sufficient condition on $G$ (Theorem 4.4) such that each linear form $I$ on $L^{2}(G)$ which is invariant under inner automorphisms is continuous and has the form $I(f)=\frac{\alpha}{\lambda(V)} \int_{V} f d x$, where $\alpha=I\left(1_{V}\right)$. In particular (Corollary 4.5 and 4.6) if $G$ is the free group on two generators or a discrete group with Kazhdan's property $T$ and every nontrivial conjugacy class in $G$ is infinite (e.g., $\operatorname{SL}(n, \mathbb{Z}), n \geq 3$ ), then every inner invariant linear form on $l^{2}(G)$ is continuous. (See [18] for a discussion of similar problems.)

It is well known (see [6 or 26]) that amenability of a locally compact group $G$ may be characterized in terms of fixed points for affine maps on compact convex sets. In $\S 5$, we characterize inner amenability of $G$ in terms of a fixed point property for left Banach $G$-modules. Finally in $\S 6$, a few miscellaneous results on inner amenability are stated and proved.

The literature on inner amenability has grown substantially in recent years: see $[1,2,7,14,16,17,20,31]$.

\section{Preliminaries AND some notations}

Throughout this paper $G$ denotes a locally compact group with a fixed left Haar measure $\lambda$. The spaces $L^{p}(G), 1 \leq p \leq \infty$, of measurable functions will be as defined in [13]. For each $a \in G, 1 \leq p<\infty$, let $\pi(a)$ be the operator on $L^{p}(G)$ defined by

$$
\pi(a) f(t)=f\left(a^{-1} t a\right) \Delta^{1 / p}(a), \quad a, t \in G, f \in L^{p}(G),
$$

where $\Delta$ is the modular function on $G$. The group $G$ is called amenable if there exists a mean $m$ on $L^{\infty}(G)$ (i.e., $m \in L^{\infty}(G), m \geq 0$, and $\|m\|=1$ ) such that $m\left(l_{a} f\right)=m(f)$ for all $a \in G$ and $f \in L^{\infty}(G)$. As is well known [8, Theorem 2.2.1], this is equivalent to the existence of a left invariant mean on $U_{r}(G)$, the space of bounded right uniformly complex-valued continuous 
functions on $G$ (as defined in [13, p. 21]). All abelian groups and all compact groups are amenable. However, if $G$ contains the free group on two generators as a closed subgroup (e.g., if $G=\operatorname{SL}(2, \mathbb{R})$ ), then $G$ is not amenable (see [8, 21, 23] for details).

Let $\mathscr{M}$ be a von Neumann algebra on a Hilbert space $H$. If $\mathscr{M}$ has property $P$, then there exists a projection of norm one $E$ from $\mathscr{B}(H)$ onto $\mathscr{M}^{\prime}$ with $E(1)=1$ (see $[28$, p. 207 , or 24, p. 136]). Von Neumann algebras with this latter property are called injective. As is well known [4], injectivity and property $P$ are equivalent. For a discussion of the various forms of amenability for von Neumann algebras, see [21, 2.35].

If $X$ is a subset of a locally convex space $E$ with topology $\tau$, then $\overline{\mathrm{co}}^{\tau} X$ will denote the closed convex hull of $X$ in $E$.

\section{INNER AMENABILITY, AMENABILITY, AND INJECTIVITY}

A reference for the definitions below in the discrete cases is Zimmer [32]. Let $X$ be a locally compact Hausdorff space. Let $G$ act invertibly on $X$ on the right such that the mapping $X \times G \rightarrow X$ defined by $(x, g) \rightarrow x \cdot g, x \in X$, $g \in G$, is jointly continuous. Let $\mu$ be a nonnegative quasi-invariant Radon measure on $X$. We define $L^{p}(X \times G, \mu \times \lambda)$ or simply $L^{p}(X \times G), 1 \leq p \leq \infty$, as the usual $L^{p}$-spaces of Borel functions identified when they coincide off a locally $(\mu \times \lambda)$-null set in $X \times G$. For each $a \in G$, define $\mu_{a}(E)=\mu(E a)$. Then, by quasi-invariance of $\mu$, we have $\mu_{a} \ll \mu$ for each $a \in G$ and there is, by the Radon-Nikodým theorem, a locally $\mu$-integrable function $r(\cdot, a)$ such that

$$
\int f\left(x a^{-1}\right) d \mu(x)=\int f(x) r(x, a) d \mu(x)
$$

for all $f \in L^{1}(X) \quad\left(=L^{1}(X, \mu)\right)$. It follows that $r(x, a b)=r(x, a) r(x a, b)$ for $a, b \in G$, and $r(x, e)=1$. For $u \in G$ and $\phi \in L^{\infty}(X)$, define the operators $U_{a}, V_{a}, M_{\phi}$, and $N_{\phi}$ on $L^{2}(X \times G)$ by

$$
\begin{aligned}
& U_{a} f(x, b)=f(x a, b a) r(x, a)^{1 / 2} \Delta(a)^{1 / 2}, \\
& V_{a} f(x, b)=f\left(x, a^{-1} b\right), \\
& M_{\phi} f(x, b)=\phi(x) f(x, b), \\
& N_{\phi} f(x, b)=\phi\left(x b^{-1}\right) f(x, b),
\end{aligned}
$$

where $f \in L^{2}(X \times G)$.

Then each $U_{a}, V_{a}$ is a unitary operator on $L^{2}(X \times G)$. Let $\mathscr{L}$ be the von Neumann algebra generated by the operators $V_{a}, N_{\phi}\left(a \in G, \phi \in L_{\infty}(X)\right)$, and $\mathscr{R}$ be the von Neumann algebra generated by the operators $U_{a}, M_{\phi}(a \in G$, $\left.\phi \in L_{\infty}(X)\right)$. If $J \in \mathscr{B}\left(L^{2}(X \times G)\right)$ is given by

$$
(J f)(x, b)=f\left(x b^{-1}, b^{-1}\right) r\left(x, b^{-1}\right)^{1 / 2} \Delta\left(b^{-1}\right)^{1 / 2},
$$


then $J^{2} f=f, J V_{a} J=U_{a}$, and $J N_{\phi} J=M_{\phi}$. So $J$ implements a spatial isomorphism between $\mathscr{L}$ and $\mathscr{R}$. Therefore $\mathscr{L}$ has property $P$ if and only if $\mathscr{R}$ has property $P$.

If $a \in G$, let $\delta_{a}$ denote the Dirac measure on $G$ concentrated at $a$. For any $f \in L^{\infty}(X)$, the function $\left(\delta_{a} \square f\right)(x)=f(x a)$ is defined $\mu$-locally almost everywhere on $X$ (see [10, Lemma 2.1]). Furthermore, $\delta_{a} \square f \in L^{\infty}(X)$. A linear functional $m$ on $L^{\infty}(X)$ is called a mean if $m(1)=1$ and $m(f) \geq 0$ whenever $f \geq 0$. A mean $m$ is $G$-invariant if $m\left(\delta_{g} \square f\right)=m(f)$ for all $g \in G$.

Also for any $\phi \in L^{1}(X)$ and $x \in G$, let $\delta_{x} * \phi \in L^{1}(X)$ be defined by

$$
\delta_{x} * \phi(\xi)=\left(\frac{d \mu_{x}}{d \mu}\right)(\xi) \phi\left(x^{-1} \xi\right) \quad \mu \text {-a.e. on } X
$$

(see [10, Lemma 2.2]), where $\mu_{x}(E)=\mu\left(x^{-1} E\right)$.

Theorem 3.1 below, in the special case where $X$ is a singleton, is proved in $[21$, p. 85$]$.

Theorem 3.1. Let $G, X$, and $\mathscr{L}$ be as above. Then the following are equivalent:

(a) $G$ is amenable.

(b) $\mathscr{L}$ is injective, $L^{\infty}(X)$ has a G-invariant mean, and $G$ is inner amenable.

Proof. (a) $\Rightarrow$ (b) If $G$ is amenable, then $G$ is inner amenable since every invariant mean on $G$ is inner invariant. It follows from [10, Theorem 3.1] that $L^{\infty}(X)$ has a $G$-invariant mean.

To see that $\mathscr{L}$ is injective, we first note that the von Neumann algebra generated by $\left\{N_{\phi} ; \phi \in L^{\infty}(X)\right\}$ has property $P$ (by the Markov-Katutani fixed point theorem). Hence $\mathscr{D}=\left\{N_{\phi} ; \phi \in L^{\infty}(X)\right\}^{\prime}$ is injective [28, Proposition 4.4.15]. (In fact, $\mathscr{D}$ is the von Neumann algebra generated by the $N_{\phi}$.) It suffices to show that there is a norm one projection from $\mathscr{D}$ onto $\mathscr{L}^{\prime}=\left\{V_{a} ; a \in G\right\}^{\prime} \cap \mathscr{D}$. For then $\mathscr{L}^{\prime}$ is injective and so $\mathscr{L}$ is also injective.

Let $T \in \mathscr{D}$ and $a \in G$. Then $V_{a^{-1}} T V_{a} \in \mathscr{D}$. Indeed $\mathscr{D}$ is generated by the $N_{\phi}$ 's; hence we may assume $T=N_{\phi}$. If $x \in X, b \in G$, and $f \in L^{2}(X \times G)$, we have

$$
\begin{aligned}
\left(V_{a^{-1}} N_{\phi} V_{a} f\right)(x, b) & =\left(N_{\phi} V_{a} f\right)(x, a b) \\
& =\phi\left(x b^{-1} a^{-1}\right)\left(V_{a} f\right)(x, a b)=\left(N_{a^{-1} \phi} f\right)(x, b),
\end{aligned}
$$

i.e., $V_{a^{-1}} N_{\phi} V_{a}=N_{a^{-1} \phi} \in D$. The result follows.

Let $K_{T}$ denote the $\overline{\mathrm{co}}^{w^{*}}\left\{V_{a^{-1}} T V_{a}: a \in G\right\} \quad\left(w^{*}=\right.$ weak $\left.^{*}\right)$. Then $K_{T}$ is a $w^{*}$-compact convex subset of $\mathscr{D}$. Consider the action of $G$ on $K_{T}$ defined by

$$
(a, S) \rightarrow V_{a^{-1}} S V_{a} \text {. }
$$

Then the action is separately continuous in the weak operator topology WOT, which agrees with the $w^{*}$-topology on $K_{T}$. Indeed, if $a_{\alpha} \rightarrow a_{0}$ and $S \in K_{T}$, 
then $V_{a_{\alpha}} \rightarrow V_{a}$ and $V_{a_{\alpha}^{-1}} \rightarrow V_{a^{-1}}$ in the strong operator topology (SOT). In particular, $S V_{a_{\alpha}} \rightarrow S V_{a}^{\alpha}$ in the SOT, and so $V_{a_{\alpha}^{-1}} S V_{a_{\alpha}} \rightarrow V_{a^{-1}} S V_{a}$ in the SOT (since multiplication is jointly continuous on bounded sets in the SOT). Hence $V_{a_{\alpha}^{-1}} S V_{a_{\alpha}} \rightarrow V_{a^{-1}} S V_{a}$ in the WOT. Now if $a \in G$, and $S_{\alpha} \rightarrow S$ in the WOT, then for any $\eta, \xi \in L_{2}(G \times X)$,

$$
\left\langle V_{a^{-1}} S_{\alpha} V_{a} \xi, n\right\rangle=\left\langle S_{\alpha} V_{a} \xi, V_{a} \eta\right\rangle \rightarrow\left\langle S V_{a} \xi, V_{a} \eta\right\rangle=\left\langle V_{a^{-1}} S V_{a} \xi, \eta\right\rangle,
$$

i.e., $V_{a^{-1}} S_{\alpha} V_{a} \rightarrow V_{a^{-1}} S V_{a}$ in the WOT. Apply now Rickert's generalization of Day's fixed point theorem to obtain $S \in K_{T}$ such that $V_{a^{-1}} S V_{a}=S$ for all $a \in S$, i.e., $S V_{a}=V_{a}$ for all $a \in S$. So $S \in\left\{V_{a}: a \in S\right\}^{\prime} \cap \mathscr{D}$. Consequently, there exists a projection $Q: \mathscr{D} \rightarrow\left\{V_{a}: a \in G\right\}^{\prime} \cap \mathscr{D}$ such that $Q(T) \in K_{T}$ for all $T \in \mathscr{D}, Q(I)=I$, and $\|Q\|=1$ by Yeadon's Theorem [30].

(b) $\Rightarrow$ (a) Define a left and a right action of $G$ on $L^{\infty}(X \times G)$ by

$$
(F a)(x, b)=F(x, a b), \quad(a F)(x, b)=F(x a, b a) .
$$

Using (1) and the equalities $r(x, a b)=r(x, a) r(x a, b)$ a.e. $x$, and $r(x, e)=$ 1 for all $x \in G$, one shows that

$$
\left\langle F, V_{a} f\right\rangle=\langle F a, f\rangle, \quad\left\langle F, U_{a} f\right\rangle=\left\langle a^{-1} F, f\right\rangle
$$

$\left(F \in L^{\infty}(X \times G), f \in L^{1}(X \times G)\right)$. Here (with a slight abuse of notation),

$$
\begin{array}{r}
V_{a} f(x, b)=f\left(x, a^{-1} b\right), \quad U_{a} f(x, b)=f(x a, b a) r(x, a) \Delta(a) \\
\left(f \in L^{1}(X \times G), x \in X, a, b \in G\right) .
\end{array}
$$

We now show that there exists a positive linear functional $m^{\prime}$ with $\left\|m^{\prime}\right\|=1$ such that

$$
m^{\prime}\left(a F a^{-1}\right)=m^{\prime}(F)
$$

for all $a \in G$ and $F \in L^{\infty}(X \times F)$.

Since $L^{\infty}(X)$ has a $G$-invariant mean, an argument similar to that of Namioka [19] shows that there exists a net $\left\{\phi_{\alpha}\right\}$ in $P_{1}(X)=\left\{\phi \in L^{1}(X): \phi \geq 0\right.$ and $\left.\|\phi\|_{1}=1\right\}$ such that $\left\|\delta_{a} * \phi_{\alpha}-\phi_{\alpha}\right\| \rightarrow 0$ for each $a \in G$. Also since $G$ is inner amenable, there exists a net $\left\{\mu_{\beta}\right\}$ in $P_{1}(G)$ such that $\left\|\delta_{a} * \mu_{\beta} * \delta_{a^{-1}}-\mu_{\beta}\right\|_{1} \rightarrow$ 0 (see [17, Proposition 1]). Let

$$
m_{\alpha, \beta}(F)=\int F d\left(\phi_{\alpha} \times \mu_{\beta}\right),
$$

where $F \in L^{\infty}(X \times G)$. Then $\left\{m_{\alpha, \beta}\right\}$ is bounded in $L^{\infty}(X \times C)^{*}$. Further- 
more, if $a \in G$ and $F \in L^{\infty}(X \times G)$, then

$$
\begin{aligned}
\left|\left\langle m_{\alpha, \beta}, a F a^{-1}\right\rangle-\left\langle m_{\alpha, \beta}, F\right\rangle\right| \\
=\left|\iint F\left(x a, a^{-1} b a\right) d \phi_{\alpha}(x) d \mu_{\beta}(b)-\iint F(x, b) d \phi_{\alpha}(x) d \mu_{\beta}(b)\right| \\
\leq\left|\iint F\left(x a, a^{-1} b a\right) d \phi_{\alpha}(x) d \mu_{\beta}(b)-\iint F\left(x, a^{-1} b a\right) d \phi_{\alpha}(x) d \mu_{\beta}(b)\right| \\
+\left|\iint F\left(x, a^{-1} b a\right) d \phi_{\alpha}(x) d \mu_{\beta}(b)-\iint F(x, b) d \phi_{\alpha}(x) d \mu_{\beta}(b)\right| \\
\leq\left\|\delta_{a} * \phi_{\alpha}-\phi_{\alpha}\right\|\|F\|_{\infty}+\left\|\delta_{a} * \mu_{\beta} * \delta_{a^{-1}}-\mu_{\beta}\right\|\|F\|_{\infty}
\end{aligned}
$$

which converges to zero. Hence if $m^{\prime}$ is any weak*-cluster point of the $\left\{m_{\alpha, \beta}\right\}$, then $m^{\prime}$ satisfies (3).

By (3) and an idea of Namioka [19] there exists a net $\left\{f_{\delta}\right\}$ in $L_{1}(X \times G)$, $f_{\delta} \geq 0,\left\|f_{\delta}\right\|_{1}=1$ such that $\left\|\left(V_{a^{-1}}-U_{a}\right) b_{\delta}\right\|_{1} \rightarrow 0$. Let $g_{\delta}=f_{\delta}^{1 / 2}$. Note that $g_{\delta} \in L_{2}(X \times G), g_{\delta} \geq 0$, and $\left\|g_{\delta}\right\|_{2}=1$. Then $\left(V_{a} f_{\delta}\right)^{1 / 2}=V_{a} g_{\delta}$, $\left(U_{a} f_{\delta}\right)^{1 / 2}=U_{a} g_{\delta}$, and hence

$$
\left\|\left(V_{a^{-1}}-U_{a}\right) g_{\delta}\right\|_{2} \rightarrow 0 \text { for all } a \in G .
$$

For each $F \in L^{\infty}(X \times G)$, let $L_{F} \in \mathscr{B}\left(L_{2}(X \times G)\right)$ be defined by

$$
L_{F} f(x, b)=F(x, b) f(x, b) \text {. }
$$

Then, as readily checked,

$$
V_{a} L_{F} V_{a^{-1}}=L_{F a^{-1}}
$$

for each $a \in G$. Let $H$ denote the group of unitary elements in the von Neumann algebra $\mathscr{R}$ with the strong operator topology. Let $\psi_{\delta}$ be a function on $H$ defined by $\psi_{\delta}(F)(U)=\left\langle U L_{F} U^{*} g_{\delta}, g_{\delta}\right\rangle(U \in H)$. Then $\psi_{\delta} \in U_{r}(H)$. Also

$$
\begin{aligned}
\psi_{\delta}\left(F a^{-1}\right)(U) & =\left\langle U L_{F a^{-1}} U^{*} g_{\delta}, g_{\delta}\right\rangle \\
& =\left\langle U V_{a} L_{F} V_{a^{-1}} U^{*} g_{\delta}, g_{\delta}\right\rangle \\
& =\left\langle U L_{F} U^{*}\left(V_{a^{-1}} g_{\delta}\right), V_{a^{-1}} g_{\delta}\right\rangle
\end{aligned}
$$

using (5) and the fact that each $V_{a}$ is in the commutant of $\mathscr{R}$. Also

$$
\psi_{\delta}(F) V_{a^{-1}}(U)=\left\langle U L_{F} U^{*}\left(V_{a} g_{\delta}\right), V_{a} g_{\delta}\right\rangle \text {. }
$$

So

$$
\begin{aligned}
& \left|\left[\psi_{\delta}\left(F a^{-1}\right)-\psi_{\delta}(F) U_{a^{-1}}\right](U)\right| \\
& =\left|\left\langle U L_{F} U^{*} V_{a^{-1}} g_{\delta}, V_{a^{-1}} g_{\delta}\right\rangle-\left\langle U L_{F} U^{*} V_{a} g_{\delta}, V_{a} g_{\delta}\right\rangle\right| \\
& =\mid\left\langle U L_{F} U^{*}\left(V_{a^{-1}}-V_{a}\right) g_{\delta}, V_{a} g_{\delta}\right\rangle \\
& +\left\langle U L_{F} U^{*} V_{a^{-1}} g_{\delta},\left(V_{a^{-1}}-V_{a}\right) g_{\delta}\right\rangle \mid \\
& \leq 2\|F\|\left\|V_{a^{-1}}-V_{a}\right\|\left\|g_{\delta}\right\|_{2} \text {. }
\end{aligned}
$$


Since $\mathscr{L}$ is injective, $\mathscr{L}$ must have property $P$. So $\mathscr{R}$ also has property $P$. By a result of de la Harpe [12], there exists a left invariant mean $m$ on $U_{r}(H)$, the space of bounded right uniformly continuous functions on $H$. Hence using (4) and (7), we have

$$
\left|m\left(\psi_{\delta}\left(F a^{-1}\right)\right)-m\left(\psi_{\delta}(F)\right)\right| \rightarrow 0 .
$$

Let $n_{\delta}=m \circ \psi_{\delta}$. Then $n_{\delta}$ is a mean on $L^{\infty}(X \times G)$. Let $n$ be a weak*-cluster point of $\left\{n_{\delta}\right\}$. Then $n\left(F a^{-1}\right)=n(F)$ for all $F \in L^{\infty}(X \times G)$ and $a \in G$. Define

$$
\tilde{n}(\phi)=n(1 \otimes \phi), \quad \phi \in L^{\infty}(G) .
$$

Then $\tilde{n}$ is a left invariant mean on $L^{\infty}(G)$. Hence $G$ is amenable.

A well-known result of Schwartz [29] asserts that if $G$ is discrete then $G$ is amenable if and only if $\operatorname{VN}(G)$ has property $P$. Letting $G$ act trivially on a set consisting of one point, we obtain from Theorem 3.1 the following [21, 2.35]:

Corollary 3.2. Let $G$ be a locally compact group. The following are equivalent:

(a) $G$ is amenable.

(b) $\operatorname{VN}(G)$ is injective and $G$ is inner amenable.

Corollary 3.3. Let $G$ be an $[I N]$-group. Then $\operatorname{VN}(G)$ is injective if and only if $G$ is amenable.

Corollary 3.4 (Losert and Rindler [17]). Let $G$ be a connected locally compact group. Then $G$ is amenable if and only if $G$ is inner amenable.

Proof. If $G$ is inner amenable, let $U$ be a compact neighborhood of $G$. Then $G_{0}=\bigcup_{n=1}^{\infty} U^{n}$ is an open (and hence closed), compactly generated subgroup of $G$. Since $G$ is connected, $G=G_{0}$. Let $K$ be a compact normal subgroup such that $G / K$ is separable metrizable (see [13, p. 71]). Clearly $G / K$ is connected and inner amenable (Proposition 6.2). However $\mathrm{VN}(G / K)$ is injective [5, p. 112 ]. So $G / K$ is amenable by Theorem 3.1 . Hence $G$ is also amenable.

\section{4. [IN]-GROUPS AND INNER AMENABILITY}

Let $G$ be an [IN]-group. Then there exists a compact neighborhood $V$ of $e$ such that $x^{-1} V x=V$ for each $x \in G$. In this section we find necessary conditions such that there exists an inner invariant mean $m$ on $L^{\infty}(G)$ with $m\left(1_{V}\right)=0$. We first establish the following general lemma.

Lemma 4.1. Let $G$ be a locally compact group. Let $\{\pi, H\}$ be a continuous unitary representation of $G$. Let $\eta_{0} \in H, \eta_{0} \neq 0$, and $\pi(x) \eta_{0}=\eta_{0}$ for all $x \in G$. Let $H_{0}=\left\{\eta \in H ;\left\langle\eta, \eta_{0}\right\rangle=0\right\}$ and $Q \in \mathscr{B}(H)$ be defined by $Q(\eta)=\left\langle\eta, \eta_{0}\right\rangle \eta_{0} /\left\|\eta_{0}\right\|^{2}$. The following are equivalent:

(a) $Q \notin C_{\pi}^{*}(G)$ (the $C^{*}$-algebra generated by $\{\pi(x) ; x \in G\}$ ). 
(b) There exists a net $\theta_{\alpha} \in H_{0}$ such that $\left\|\theta_{\alpha}\right\|=1$, and $\left\|\pi(x) \theta_{\alpha}-\theta_{\alpha}\right\| \rightarrow 0$ for each $x \in G$.

(c) There exists a state $\omega$ on $\mathscr{B}(H)$ such that $\omega(\pi(x))=1$ for each $x \in G$ and $\omega(Q)=0$.

Proof. (a) $\Rightarrow$ (b) We follow an idea contained in the proof of [3, Theorem 1.1]. Suppose (b) fails; then we can find $y_{1}, \ldots, y_{M} \in G$ and $\varepsilon>0$, such that for all $\theta \in H_{0},\|\theta\|=1$, there exists some $i, 1 \leq i \leq M$, such that $\left.\| \pi\left(y_{i}\right) \theta-\theta\right) \| \geq \varepsilon$. Let $x_{1}=e$, the identity of $G$, and $x_{2}=y_{1}, \ldots, x_{M+1}=y_{M}$. Let $N=M+1$ and $A=N^{-1} \sum_{k=1}^{N} \pi\left(x_{k}\right)$. We claim that $\|A\|_{H_{0}}<1$. If not, we can find a sequence $\theta_{n} \in H_{0},\left\|\theta_{n}\right\|=1$, such that

$$
\left\|A\left(\theta_{n}\right)\right\|_{2}^{2}=\left\langle A\left(\theta_{n}\right), A\left(\theta_{n}\right)\right\rangle=\frac{1}{N^{2}} \sum_{i, j}\left\langle\pi\left(x_{j}^{-1} x_{i}\right) \theta_{n}, \theta_{n}\right\rangle \rightarrow 1 .
$$

Since $\left|\left\langle\pi\left(x_{j}^{-1} x_{i}\right) \theta_{n}, \theta_{n}\right\rangle\right| \leq 1$ for each $i, j$, we conclude that

$$
\operatorname{Re}\left\langle\pi\left(x_{j}^{-1} x_{i}\right) \theta_{n}, \theta_{n}\right\rangle \rightarrow 1 .
$$

But then

$$
\left\|\pi\left(x_{i}\right) \theta_{n}-\pi\left(x_{j}\right) \theta_{n}\right\|_{2}^{2}=2-\operatorname{Re}\left\langle\pi\left(x_{j}^{-1} x_{i}\right) \theta_{n}, \theta_{n}\right\rangle \rightarrow 0
$$

as $n \rightarrow \infty$. In particular, since $x_{1}=e$ and $x_{k+1}=y_{k}, k=1, \ldots, M$, we conclude that

$$
\lim _{n}\left\|\pi\left(y_{k}\right) \theta_{n}-\theta_{n}\right\|_{2}=0 \text { for each } k, 1 \leq k \leq m .
$$

This contradicts the choice of $y, \ldots, y_{M}$. Thus $\|A\|_{H_{0}}<1$ as claimed.

Observe now that if $\eta \in H$, then

(1) $Q(\eta)=A^{m}(Q(\eta))$. Indeed, if $x \in G$, then

$$
\begin{aligned}
\pi(x) Q(\eta) & =\frac{1}{\left\|\eta_{0}\right\|^{2}}\left\langle\eta, \eta_{0}\right\rangle \pi(x)\left(\eta_{0}\right) \\
& =\frac{1}{\left\|\eta_{0}\right\|^{2}}\left\langle\eta, \eta_{0}\right\rangle \eta_{0}=Q(\eta)
\end{aligned}
$$

by the invariance of $\eta_{0}$.

(2) $\eta-Q(\eta) \in H_{0}$. Indeed,

$$
\left\langle\eta-Q(\eta), \eta_{0}\right\rangle=\left\langle\eta, \eta_{0}\right\rangle-\frac{1}{\left\|\eta_{0}\right\|^{2}}\left\langle\eta, \eta_{0}\right\rangle\left\langle\eta_{0}, \eta_{0}\right\rangle=0 .
$$

Hence we have for $m$ fixed and $\eta \in H$,

$$
\begin{aligned}
\left\|\left(A^{m}-Q\right) \eta\right\|_{2} & =\left\|A^{m}(\eta-Q \eta)\right\| \quad(\text { by }(1)) \\
& \leq\left\|A^{m}\right\|_{H_{0}}\|\eta-Q \eta\| \quad(\text { by }(2)) \\
& \leq 2\|A\|_{H_{0}}^{m}\|\eta\| . \\
\therefore\left\|A^{m}-Q\right\| & \leq 2\|A\|_{H_{0}}^{m} \rightarrow 0, \quad \text { i.e., } Q \in C_{\pi}^{*}(G) .
\end{aligned}
$$


(b) $\Rightarrow$ (c) Let $\omega_{\alpha}=\left\langle T \theta_{\alpha}, \theta_{\alpha}\right\rangle$ and $\omega$ be a weak*-cluster point of $\left\{\omega_{\alpha}\right\}$ in $\mathscr{B}(H)$. Then clearly $\omega(\pi(x))=1$ for each $x \in G$, and $\omega(Q)=0$.

(c) $\Rightarrow$ (a) If $X=\sum_{i=1}^{n} \lambda_{i}\left(x_{i}\right)$, then

$$
\|X-Q\| \geq|\omega(X)-\omega(Q)|=\left|\sum_{i=1}^{n} \lambda_{i}\right|
$$

and

$$
\begin{aligned}
\|X-Q\| & \geq|\langle(X-Q) \theta, \theta\rangle| \quad\left(\text { where } \theta=\frac{\eta_{0}}{\left\|\eta_{0}\right\|}\right) \\
& =|\langle X \theta, \theta\rangle-\langle Q \theta, \theta\rangle| \\
& =\left|\frac{1}{\left\|\eta_{0}\right\|^{2}}\left\langle\sum \lambda_{i} \eta_{0}, \eta_{0}\right\rangle-\left\langle\frac{1}{\left\|\eta_{0}\right\|^{2}}\left\langle\frac{\eta_{0}}{\left\|\eta_{0}\right\|}, \eta_{0}\right\rangle \eta_{0}, \frac{\eta_{0}}{\left\|\eta_{0}\right\|}\right\rangle\right| \\
& =\left|\sum \lambda_{i}-\frac{1}{\left\|\eta_{0}\right\|^{2}} \cdot \frac{1}{\left\|\eta_{0}\right\|^{2}}\left\langle\eta_{0}, \eta_{0}\right\rangle\left\langle\eta_{0}, \eta_{0}\right\rangle\right| \\
& =\left|\sum \lambda_{i}-1\right| .
\end{aligned}
$$

Hence $\|X-Q\| \geq \max \left\{\left|\sum x_{i}\right|,\left|1-\sum \lambda_{i}\right|\right\} \geq \frac{1}{2} . \therefore Q \notin C_{\pi}^{*}(G)$.

For each $x \in G$, let $\pi(x) f(t)=f\left(x^{-1} t x\right) \Delta(x)^{1 / 2}, t \in G, f \in L^{2}(G)$. Then $\left\{\pi, L^{2}(G)\right\}$ is a continuous unitary representation of $G$. Let $C_{\pi}^{*}(G)$ denote the $C^{*}$-algebra generated by $\{\pi(x) ; x \in G\}$ in $\mathscr{B}\left(L^{2}(G)\right)$. A discrete version of the following result is proved in [20].

Theorem 4.2. Let $G$ be a locally compact group and $V$ be a compact neighborhood of $e$ such that $x^{-1} V x=V$ for all $x \in G$. Let $L_{0}^{2}(V)=\{g \in$ $\left.L^{2}(G) ; \int_{V} g(x) d x=0\right\}$. Consider the following conditions on $G$ :

(a) The operator $Q_{V}(f)=\frac{1}{\lambda(V)} \int_{V} f(x) d x \cdot 1_{V}$ is not in $C_{\pi}^{*}(G)$.

(b) There exists a net $\left\{h_{\alpha}\right\}$ in $L_{0}^{2}(V)$ such that $\left\|h_{\alpha}\right\|_{2}=1$ and

$$
\left\|\pi(x) h_{\alpha}-h_{\alpha}\right\|_{2} \rightarrow 0 \text { for each } x \in G \text {. }
$$

(c) There exists a state $\omega$ on $\mathscr{B}(H)$ such that $\omega(\pi(x))=1$ for each $x \in G$, and $\omega(Q)=0$.

(d) There exists an inner invariant mean $m$ on $L^{\infty}(G)$ such that $m\left(l_{V}\right)=$ 0 .

Then $(\mathrm{a}) \Leftrightarrow(\mathrm{b}) \Leftrightarrow(\mathrm{c}) \Leftarrow(\mathrm{d})$.

Proof. That $(\mathrm{a}) \Leftrightarrow(\mathrm{b}) \Leftrightarrow$ (c) follows from Lemma 4.1.

(d) $\Rightarrow$ (b) Indeed, as in Losert and Rindler, there exists a net $\nu_{\alpha} \in L^{1}(G)$, $\nu_{\alpha} \geq 0,\left\|\nu_{\alpha}\right\|_{1}=1, \nu_{\alpha}(V)=0$, and $\left\|\pi(x) \nu_{\alpha}\right\|_{1} \rightarrow 0$. Let $h_{\alpha}=\nu_{\alpha}^{1 / 2}$; then $\left\|\pi(x) h_{\alpha}-h_{\alpha}\right\|_{1} \rightarrow 0$ for all $x \in G,\left\|h_{\alpha}\right\|_{2}=1$. Furthermore,

$$
\left|\int_{V} h_{\alpha} d x\right|=\left\langle h_{\alpha} 1_{V}, 1_{V}\right\rangle \leq\left(\int_{V} h_{\alpha}^{2} d x\right)^{1 / 2} \lambda(V)^{1 / 2}=0,
$$

i.e., $h_{\alpha} \in L_{0}^{2}(V)$. 
Open Problem. Is (d) equivalent to the other conditions in Theorem 4.2? (This is the case when $G$ is discrete and $V=\{e\}$ as shown in [20].)

Lemma 4.3. Let $G,\{\pi, H\}, \eta_{0}, H_{0}$, and $Q$ be as in Lemma 4.1. If $Q \in$ $C_{\pi}^{*}(G)$, then each linear form $I$ on $H$ which in invariant under $\{\pi(x): x \in G\}$ is continuous, and has the form

$$
I(\eta)=\frac{\alpha}{\left\|\eta_{0}\right\|^{2}}\left\langle\eta, \eta_{0}\right\rangle, \quad \text { where } \alpha=I\left(\eta_{0}\right) .
$$

Proof. As in the proof of Lemma 4.1, (a) $\Rightarrow(\mathrm{b})$, there exists $x_{1}, \ldots, x_{N+1} \in$ $G$, such that $x_{1}=e$, and the operator $A=(N+1)^{-1} \sum_{k=1}^{N+1} \pi\left(x_{k}\right)$ satisfies $\|A\|_{H_{0}}<1$. In particular, for each $\theta_{0} \in H_{0}$, the series $\theta=\sum_{n=0}^{\infty} A^{n}\left(\theta_{0}\right)$ converges in $H_{0}$. Also,

$$
\begin{aligned}
\theta_{0} & =\theta-A \theta=\frac{N \theta}{N+1}-\frac{1}{N+1} \sum_{i=2}^{N+1} \pi\left(x_{i}\right) \theta \\
& =\sum_{i=2}^{N+1}\left(\gamma-\pi\left(x_{i}\right) \gamma\right) \quad \text { with } \gamma=\frac{\theta}{N+1}
\end{aligned}
$$

Let $\eta \in H$; then $\theta_{0}=\eta-Q(\eta) \in H_{0}$. So

$$
\eta=\eta-Q(\eta)+Q(\eta)=\sum_{i=1}^{n}\left(\gamma-\pi\left(x_{i}\right) \gamma\right)+Q(\eta) .
$$

So if $I$ is invariant on $H$, then

$$
I(\eta)=I(Q(\eta))=\frac{1}{\left\|\eta_{0}\right\|^{2}}\left\langle\eta, \eta_{0}\right\rangle I\left(\eta_{0}\right) .
$$

The following is an analogue of the main result in [27].

Theorem 4.4. Let $G$ be a locally compact group and $V$ be a neighborhood of $e$ such that $x^{-1} V x=V$ for all $x \in G, 0<\lambda(V)<\infty$. If $Q_{V} \in C_{\pi}^{*}(G)$, then each linear form $I$ on $L^{2}(G)$ which is invariant under inner automorphism is continuous and has the form

$$
I(f)=\frac{\alpha}{\lambda(V)} \cdot \int_{V} f d x, \quad \text { where } \alpha=I\left(1_{V}\right) .
$$

Proof. This follows from Lemma 4.3.

Corollary 4.5. Let $G$ be an I.C.C. discrete group with Kazhdan's property $T$. Then every inner invariant linear form on $L^{2}(G)$ is continuous.

Proof. In this case $\delta_{e}$ is the only inner invariant mean on $L^{\infty}(G)$. By Paschke's Theorem [20], $Q_{V} \in C_{\pi}^{*}(G)$ when $V=\{e\}$. Apply Theorem 4.4.

Corollary 4.6. Let $G$ be the free group on two generators. Then every inner invariant form on $L^{2}(G)$ is continuous.

Proof. By the result of Effros [7], $\delta_{e}$ is the only inner invariant mean on $L^{\infty}(G)$. Apply now Paschke's Theorem [20] and Theorem 4.4. 
Let $V$ be a measurable subset of a locally compact group $G$. Let $L^{2}(V)=$ $\left\{f \in L_{2}(G): f_{\left.\right|_{V}}=0\right\}$. Then $L^{2}(V)$ is a closed subspace of $L_{2}(G)$ and $L^{2}(G)=$ $L^{2}(V) \oplus L^{2}(G \sim V)$. Let $P_{V}$ be the orthogonal projection of $L^{2}(V)$.

Proposition 4.7. Let $G$ be a locally compact group. Let $V$ be a measurable subset of $G$ such that $x V x^{-1}=V$ for all $x \in G$. Suppose there exist inner invariant means $m, n$ such that $m(V)=0$ and $n(G \sim V)=0$. Then $\left\|T-P_{A}\right\| \geq \frac{1}{2}$ for each $T \in C_{\pi}^{*}(G)$.

Proof. Using an idea of Namioka [19], we may find nets $\left\{f_{\delta}\right\}$ and $\left\{g_{\alpha}\right\}$ of positive norm one functions in $L^{1}(G)$ such that $f_{\delta}(A)=0, g_{\alpha}(G \sim A)=0$, $\left\|\pi(x) f_{\delta}-f_{\delta}\right\|_{1} \rightarrow 0$, and $\left\|\pi(x) g_{\alpha}-g_{\alpha}\right\|_{1} \rightarrow 0$ (here $\pi(x) f(t)=f\left(x^{-1} t x\right) \Delta(x)$, $\left.f \in L_{1}(G) \quad(x, t \in G)\right)$. Let $f_{\delta}^{\prime}=f_{\delta}^{1 / 2}$ and $g_{\alpha}^{\prime}=g_{\alpha}^{1 / 2}$. Then $f_{\delta}^{\prime}$ and $g_{\alpha}^{\prime}$ are positive norm one functions in $L^{2}(G), f_{\delta}^{\prime}(A)=0, g_{\alpha}^{\prime}(G \sim A)=0$, $\left\|\pi(x) f_{\delta}^{\prime}-f_{\delta}^{\prime}\right\|_{2} \rightarrow 0$, and $\left\|\pi(x) g_{\alpha}^{\prime}-g_{\alpha}^{\prime}\right\|_{2} \rightarrow 0$. Let $x_{1}, \ldots, x_{n} \in G, \alpha_{1}, \ldots, \alpha_{n}$ $\in \mathbb{C}$, and $T=\sum_{i=1}^{n} \alpha_{i} \pi\left(x_{i}\right)$. Then

$$
\begin{aligned}
\left\|T-P_{A}\right\| & \geq \underset{\delta}{\limsup }\left\|T f_{\delta}-P_{A} f_{\delta}\right\|_{2} \\
& =\underset{\delta}{\limsup }\left\|\sum_{i=1}^{n} \alpha_{i} \pi\left(x_{i}\right) f_{\delta}\right\|_{2}=\left|\sum_{i=1}^{n} \alpha_{i}\right| .
\end{aligned}
$$

Also

Hence

$$
\begin{aligned}
& \left\|T-P_{A}\right\| \geq \underset{\alpha}{\limsup }\left\|T g_{\alpha}-P_{A} g_{\alpha}\right\|_{2} \\
& =\underset{\alpha}{\limsup }\left\|\sum_{i=1}^{n} \alpha_{i}\left(\pi\left(x_{i}\right) g_{\alpha}-g_{\alpha}\right)+\left(\sum_{i=1}^{n} \alpha_{i}-1\right) g_{\alpha}\right\| \\
& =\left|\sum_{i=1}^{n} \alpha_{i}-1\right| \text {. }
\end{aligned}
$$

$$
\left\|T-P_{A}\right\| \geq \max \left\{\left|\sum_{i=1}^{n} \alpha_{i}\right|,\left|\sum_{i=1}^{n} \alpha_{i}-1\right|\right\} \geq \frac{1}{2} .
$$

\section{A FIXED POINT PROPERTY}

Let $G$ be a locally compact group. A left Banach $G$-module $X$ is a Banach space $X$ which is a left $G$-module such that

(i) $\|a \cdot x\| \leq\|x\|$ for all $x \in X$ and $a \in G$.

(ii) For all $x \in X$, the map $a \rightarrow a \cdot x$ is continuous from $G$ into $X$.

In this case, we define $\langle f \cdot a, x\rangle=\langle f, a \cdot x\rangle$ for each $f \in X^{*}, a \in G$, and $x \in X$.

If $\mu \in M(G)$ and $f \in X^{*}$, we define

$$
\langle f \cdot \mu, x\rangle=\int\langle f, a \cdot x\rangle d \mu(a), \quad x \in X .
$$


Then $f \cdot \mu \in X^{*}, f \cdot \mu=f \cdot a$ if $\mu=\delta_{a}$, and $\left(f \cdot \mu_{1}\right) \cdot \mu_{2}=f \cdot\left(\mu_{1} * \mu_{2}\right)$ for $\mu_{1}, \mu_{2} \in M(G)$. Finally if $a \in G, \mu \in M(G)$, and $m \in X^{* *}$, we also define

$$
\langle a \cdot m, f\rangle=\langle m, f \cdot \alpha\rangle \text { and }\langle\mu \cdot m, f\rangle=\langle m, f \cdot \mu\rangle
$$

for all $f \in X^{*}$.

By the weak* operator topology $\left(\mathrm{W}^{*} \mathrm{OT}\right)$ on $\mathscr{B}\left(X^{* *}\right)$, we shall mean the weak* topology of $\mathscr{B}\left(X^{* *}\right)$ when it is identified with the dual space $\left(X^{* *} \otimes X^{*}\right)^{*}$ in the obvious way. This topology is determined by the seminorms $\left\{P_{f, m} ; f \in\right.$ $\left.X^{*}, m \in X^{* *}\right\}$ where $p_{f, m}(T)=|\langle T m, f\rangle|$. Of course, the unit ball in $\mathscr{B}\left(X^{* *}\right)$ is compact in the $\mathrm{W}^{*} \mathrm{OT}$.

For each $\phi \in L^{1}(G)$, let $T_{\phi} \in \mathscr{B}\left(X^{* *}\right)$ be defined by $T_{\phi}(m)=\phi \cdot m$, $m \in X^{* *}$. Let $\mathscr{P}_{X^{* *}}$ denote the closure of $\left\{T_{\phi} ; \phi \geq 0,\|\phi\|_{1}=1\right\}$ in the $\mathrm{W}^{*} \mathrm{OT}$. Then $\mathscr{P}_{X^{* *}}$ with the $\mathrm{W}^{*} \mathrm{OT}$ is compact and convex. Also if $a \in G$, let $T_{a} \in \mathscr{B}\left(X^{* *}\right)$ be defined by $T_{a}(m)=a \cdot m, m \in X^{* *}$. Inner amenability can be characterized by the following "fixed point property".

Theorem 5.1. Let $G$ be a locally compact group. The following are equivalent:

(a) $G$ is inner amenable.

(b) Whenever $X$ is a left Banach G-module there exists $T \in \mathscr{P}_{X}$ *. such that $T_{a} T=T T_{a}$ for all $a \in G$.

Proof. (a) $\Rightarrow$ (b) Let $\left\{\phi_{\alpha}\right\}$ be a net in $L^{1}(G), \phi_{\alpha} \geq 0,\left\|\phi_{\alpha}\right\|_{1}=0$, such that $\left\|\delta_{a} * \phi_{\alpha}-\phi_{\alpha} * \delta_{a}\right\|_{1} \rightarrow 0$ for each $a \in G$ [17, Proposition 1]. Since $\left\{T_{\phi_{\alpha}}\right\}$ is contained in the unit ball of $\mathscr{B}\left(X^{* *}\right)$ and the unit ball is compact in the $\mathrm{W}^{*} \mathrm{OT}$, we may assume by passing to a subnet if necessary that $T_{\phi_{a}} \rightarrow T$ in the $\mathrm{W}^{*} \mathrm{OT}, T \in \mathscr{B}\left(X^{* *}\right)$ and $\|T\| \leq 1$. Now if $a \in G$ and $m \in X^{* *}$, then

$$
\begin{aligned}
\left\|T_{a} T_{\phi_{\alpha}} m-T_{\phi_{\alpha}} T_{a} m\right\| & =T_{\delta_{a^{*} \phi_{\alpha}}}(m)-T_{\phi_{\alpha} * \delta_{a}}(m) \| \\
& \leq\left\|\delta_{a} * \phi_{\alpha}-\phi_{\alpha} * \delta_{a}\right\|_{1}\|m\| \rightarrow 0 .
\end{aligned}
$$

On the other hand, $T_{a} T_{\phi_{\alpha}} \rightarrow T_{a} T$ and $T_{\phi_{\alpha}} T_{\alpha} \rightarrow T T_{a}$ in the $\mathrm{W}^{*} \mathrm{OT}$. In particular $T_{a} T=T T_{a}$.

(b) $\Rightarrow$ (a) Let $X=L^{1}(G)$ and consider $L^{1}(G)$ as a left $G$-module where $a \cdot h=l_{a^{-1}} h, a \in G, h \in L^{1}(G)$. Given $m \in L^{\infty}(G)^{*}, f \in L^{\infty}(G)$, define $m_{L}(f) \in L^{\infty}(G)$ by

$$
\left\langle m_{L}(f), \phi\right\rangle=\left\langle m, \frac{1}{\Delta} \tilde{\phi} * f\right\rangle, \quad \phi \in L_{1}(G) .
$$

Define $\left\langle\widetilde{T}_{n}(m), f\right\rangle=\left\langle n, m_{L}(f)\right\rangle, n \in L^{\infty}(G)^{*}, f \in L^{\infty}(G)$. Then, as readily checked, $\widetilde{T}_{\phi}=T_{\phi}$ for each $\phi \in L^{1}(G)$. Furthermore, the map $n \rightarrow \widetilde{T}_{n}$ from $L^{\infty}(G)^{*}$ into $\mathscr{B}\left(L^{\infty}(G)^{*}\right)$ is continuous when $L^{\infty}(G)^{*}$ has the weak ${ }^{*}$-topology and $\mathscr{B}\left(L^{\infty}(G)^{*}\right)$ has the $\mathrm{W}^{*} \mathrm{OT}$. Hence

$$
\mathscr{P}_{L^{\infty}(G)^{*}}=\left\{\widetilde{T}_{n} ; n \in L^{\infty}(G)^{*}, n \geq 0, \text { and }\|n\|=1\right\} .
$$


By assumption, there exists $n \in L^{\infty}(G)^{*}, n \geq 0,\|n\|=1$, such that

$$
T_{a} \widetilde{T}_{n}=\widetilde{T}_{n} T_{a} \text { for all } a \in G .
$$

Next we observe that

$$
\left\langle\left(T_{a} m\right)_{L}(f), \phi\right\rangle=\left\langle m_{L}(f), \phi * \delta_{a^{-1}}\right\rangle
$$

for each $a \in G, m \in L^{\infty}(G)^{*}$, and $f \in L^{\infty}(G)$.

Hence if $\left\{\psi_{\alpha}\right\}$ is a bounded approximate identity of $L^{1}(G)$ and $m$ is a weak* cluster point of $\psi_{\alpha}$, then (by (2))

$$
\begin{aligned}
\left\langle T_{a}(m)_{L}(f), \phi\right\rangle & =\left\langle m_{L}(f), \phi * \delta_{a}\right\rangle=\left\langle m, \frac{1}{\Delta}\left(\phi * \delta_{a}\right)^{\sim} * f\right\rangle \\
& =\lim _{\alpha}\left\langle\psi_{\alpha}, \frac{1}{\Delta}\left(\phi * \delta_{a}\right)^{\sim} * f\right\rangle=\lim _{\alpha}\left\langle\phi * \delta_{a} * \psi_{\alpha}, f\right\rangle \\
& =\left\langle\phi * \delta_{a}, f\right\rangle=\left\langle r_{a} f, \phi\right\rangle
\end{aligned}
$$

for any $f \in L^{\infty}(G)$ and $\phi \in L^{1}(G)$, i.e.,

$$
T_{a}(m)_{L}(f)=r_{a} f
$$

Also

$$
\begin{aligned}
\left\langle T_{a} \widetilde{T}_{n}(m), f\right\rangle & =\left\langle\widetilde{T}_{n}(m), l_{a} f\right\rangle=\left\langle n \odot m, l_{a} f\right\rangle \\
& =\left\langle n, m_{L}\left(l_{a} f\right)\right\rangle=\left\langle n, l_{a} m_{L}(f)\right\rangle \\
& =\left\langle l_{a}^{*} n, f\right\rangle=\left\langle n, l_{a} f\right\rangle .
\end{aligned}
$$

Combining this with (1) and (3), we obtain that $\left\langle n, l_{a} f\right\rangle=\left\langle n, r_{a} f\right\rangle$ for any $f \in L^{\infty}(G)$ and $a \in G$, i.e., $n$ is an inner invariant mean.

\section{Miscellaneous Results}

Proposition 6.1. Let $G$ be a separable connected group. Then the following are equivalent:

(a) $G$ admits a countably additive inner invariant mean.

(b) $G$ is an $[I N]$-group.

(c) $G$ is an extension of a compact group by a vector group.

Proof. (a) $\Rightarrow$ (b) Let $B(G)=\{x \in G$ : the conjugacy class of $x$ has relatively compact closure $\}$. By [9, Theorem 1.4], there exists a layering of $G$ that terminates with the closed subgroup $B(G)$, i.e., a sequence

$$
B(G)=X_{0} \subset X_{1} \subset \cdots \subset X_{m}=G
$$

such that each $X_{k}$ is a closed subset of $G$ invariant under the inner automorphisms and every point $x \in X_{k} \sim X_{k-1}$ has a relative neighborhood in $X_{k}$ with infinitely many disjoint conjugates. Suppose that $m$ is a countably additive inner invariant mean and suppose that $m(B(G))=0$. Then $m\left(X_{k} \sim X_{k-1}\right)>0$ for some $k$. By separability, there exists a relatively open set $U$ in $X_{k} \sim X_{k-1}$ 
with $m(U)>0$ and a sequence $\left\{x_{n}\right\}$ with $\left\{x_{n} U x_{n}^{-1}\right\}$ pairwise disjoint. This contradicts $m(G)=1$. So $m(B(G))>0$, and hence $\lambda(B(G))>0$, where $\lambda$ is the left Haar measure on $G$. Consequently $B(G)$ is an open $[\mathrm{FC}]^{-}$-subgroup of $G$. In particular $G$ in an [IN]-group [15, Corollary 2.2].

That (b) $\Leftrightarrow$ (c) for connected groups is well known [11, Corollary 2.8]. Also (b) $\Rightarrow($ a) is clear.

Proposition 6.2. Let $G$ be a locally compact group and $H$ be a closed normal subgroup of $G$. If $G$ is inner amenable, then $G / H$ is also inner amenable.

Proof. Define a map $\phi: L^{\infty}(G / H) \rightarrow L^{\infty}(G)$ by $\phi(f)=f \circ \theta$, where $\theta$ is the quotient map of $G$ onto $G / H$. Then, as is well known (see [25, pp. 66 and 82]), $\rho$ is a linear isometry from $L^{\infty}(G / H)$ into the subspace $A$ of $L^{\infty}(G)$, where

$$
A=\left\{f \in L^{\infty}(G) ; r_{x} f=f \text { for all } x \in G\right\} .
$$

Furthermore $\rho(\pi(\dot{x}) f)=(\pi(x) f) \circ \theta$ for each $x \in G$, where $\dot{x}=x H$. Let $m$ be an inner invariant mean on $L^{\infty}(G)$. Define $m^{\prime}(f)=m(\rho(t)), f \in$ $L^{\infty}(G / H)$. Then, as is readily checked, $m^{\prime}$ is an inner invariant mean on $L^{\infty}(G / H)$.

\section{REFERENCES}

1. M. Choda, The factors of inner amenable groups, Math. Japon. 24 (1979), 145-152.

2. __ Effect of inner amenability on strong ergodicity, Math. Japon. 24 (1979), 235-246.

3. C. Chou, A. T. M. Lau, and J. Rosenblatt, Approximation of compact operators by sums of translations, Illinois J. Math. 29 (1985), 340-350.

4. A. Connes, Von Neumann algebras, Proc. Internat. Congr. Math., Helsinki, 1978, pp. 98109.

5. _ Classification of injective factors, Ann. of Math. (2) 104 (1976), 73-115.

6. M. M. Day, Fixed point theorems for compact convex sets, Illinois J. Math. 5 (1961), 585590.

7. E. G. Effros, Property $\Gamma$ and inner amenability, Proc. Amer. Math. Soc. 47 (1975), 483-486.

8. F. P. Greenleaf, Invariant means on topological groups and their applications, Van Nostrand, 1969.

9. F. P. Greenleaf, M. Moskowitz, and L. P. Rothschild, Unbounded conjugacy classes in Lie groups and location of central measures, Acta Math. 132 (1974), 225-243.

10. F. P. Greenleaf, Amenable actions of locally compact groups, J. Funct. Anal. 4 (1969), 295315.

11. S. Grosser and M. Moskowitz, Compactness conditions on topological groups, J. Reine Angew. Math. 246 (1971), 1-40.

12. P. de la Harpe, Moyennabilité du groupe unitaire et propriété $P$ de Schwartz des algèbres de von Neumann, Lecture Notes in Math., Springer-Verlag, 1979, pp. 220-227.

13. E. Hewitt and K. A. Ross, Abstract harmonic analysis, Vol. 1, Springer-Verlag, Berlin, 1963.

14. A. T. M. Lau and A. L. T. Paterson, Operator theoretic characterisations of [IN]-groups and inner amenability, Proc. Amer. Math. Soc. 102 (1988), 893-897.

15. J. R. Liukkonen, Dual spaces of groups with precompact conjugacy classes, Trans. Amer. Math. Soc. 180 (1973), 85-108. 
16. V. Losert and H. Rindler, Asymptotically central functions and invariant extensions of Dirac measures, in Proc. Conf. Probability Measures on Groups, Oberwolfach, 1983.

17. V. Losert and H. Rindler, Conjugate invariant means, Colloq. Math. 15 (1987), 221-225.

18. G. H. Meisters, Some problems and results on translation-invariant linear forms, in Proc. Conf. Radical Banach Algebras and Automatic Continuity (Long Beach, 1981), Lecture Notes in Math., Springer-Verlag, Berlin and New York, 1983, pp. 423-444.

19. I. Namioka, Folner's condition for amenable semigroups, Math. Scand. 15 (1964), 18-28.

20. W. L. Paschke, Inner amenability and conjugation operators, Proc. Amer. Math. Soc. 71 (1978), 117-118.

21. A. L. T. Paterson, Amenability, Mathematical Surveys and Monographs, no. 29, Amer. Math. Soc., Providence, R.I., 1988.

22. J. P. Pier, Quasi-invariance intérieure sur les groupes localement compacts, G.M.E.L. Actualitiés Mathématiques, Actes du $6^{\circ}$ Congrès du Groupement des Mathematicians d'Expression Latin, Luxembourg, 1982.

23. __ Amenable locally compact groups, Wiley, 1984.

24. __ Amenable Banach algebras, Pitman Res. Notes Math. Ser., no. 172, Longman Harlow, 1988.

25. H. Reiter, Classical harmonic analysis and locally compact groups, Oxford Mathematical Monographs, 1968.

26. N. W. Rickert, Amenable groups and groups with fixed point properties, Trans. Amer. Math. Soc. 127 (1967), 221-232.

27. J. Rosenblatt, Translation-invariant linear forms of $L_{p}(G)$, Proc. Amer. Math. Soc. 94 (1985), 226-228.

28. S. Sakai, $C^{*}$-algebras and $W^{*}$-algebras, Springer-Verlag, 1971.

29. J. T. Schwartz, $W^{*}$-algebras, Gordon and Breach, New York, 1967.

30. F. Y. Yeadon, Fixed points and amenability: a counterexample, J. Math. Anai. Appl. 45 (1974), 718-720.

31. C. K. Yuan, The existence of inner invariant means on $L^{\infty}(G)$, J. Math. Anal. Appl. 130 (1988), 514-524.

32. R. Zimmer, On the von Neumann algebra of an ergodic group action, Proc. Amer. Math. Soc. 66 (1977), 289-293.

Department of Mathematics, University of Alberta, Edmonton, Alberta, Canada T6R 1T1

Department of Mathematics, University of MississipPi, University, Mississippi 38677 